\author{
M. Volbeda \\ J. Wetterslev \\ C. Gluud \\ J. G. Zijlstra \\ I. C. C. van der Horst \\ F. Keus
}

\section{Glucocorticosteroids for sepsis: systematic review with meta-analysis and trial sequential analysis}

Received: 23 December 2014

Accepted: 27 May 2015

Published online: 23 June 2015

(C) The Author(s) 2015. This article is published with open access at Springerlink.com

Take-home message: There is no evidence for a beneficial effect on mortality of glucocorticosteroids in patients with sepsis.

Electronic supplementary material The online version of this article (doi:10.1007/s00134-015-3899-6) contains supplementary material, which is available to authorized users.

M. Volbeda (®) · J. G. Zijlstra • I. C. C. van der Horst . F. Keus Department of Critical Care, University of Groningen, University Medical Centre Groningen (UMCG), P.O. Box 30.001, 9700 RB Groningen, The Netherlands e-mail: m.volbeda@umcg.nl

Tel.: 0031-050-3612327

J. Wetterslev · C. Gluud

The Copenhagen Trial Unit (CTU), Centre of Clinical Intervention Research, Department 7812, Rigshospitalet, Copenhagen University Hospital, 2100 Copenhagen, Denmark
Abstract Introduction: Glucocorticosteroids (steroids) are widely used for sepsis patients. However, the potential benefits and harms of both high and low dose steroids remain unclear. A systematic review of randomised clinical trials with metaanalysis and trial sequential analysis (TSA) might shed light on this clinically important question.

Methods: A systematic review was conducted according to a published protocol and The Cochrane Handbook methodology including metaanalyses, TSA of randomised clinical trials, and external validity estimation (GRADE). Randomised clinical trials evaluating steroids were included for sepsis patients (systemic inflammatory response syndrome, sepsis, severe sepsis or septic shock) aged $>18$ years. Cochrane Central Register of Controlled Trials (CENTRAL), PubMed/Medline, Embase, Web of Science and Cinahl were searched until 18 February 2015. No language restrictions were applied. Primary outcomes were mortality at longest follow-up and serious adverse events. Results: A total of 35 trials randomising 4682 patients were assessed and reviewed in full text. All trials but two had high risk of bias. No statistically significant effect was found for any dose of steroids versus placebo or no intervention on mortality at maximal follow-up [relative risk (RR) 0.89 ; TSA adjusted confidence interval (CI) 0.74-1.08]. Two trials with low risk of bias also showed no statistically significant difference (random-effects model RR 0.38, $95 \%$ CI 0.06-2.42). Similar results were obtained in subgroups of trials stratified according to high $(>500 \mathrm{mg})$ or low $(\leq 500 \mathrm{mg})$ dose hydrocortisone (or equivalent) (RR 0.87; TSA-adjusted CI 0.38-1.99; and RR 0.90; TSA-adjusted CI 0.49-1.67, respectively). There were also no statistically significant effects on serious adverse events other than mortality (RR 1.02; TSA-adjusted CI $0.7-1.48$ ). The effects did not vary according to the degree of sepsis. TSA showed that many more randomised patients are needed before definitive conclusions may be drawn. Conclusion: Evidence to support or negate the use of steroids in any dose in sepsis patients is lacking. The results of ongoing and future welldesigned, large randomised clinical trials are needed.

Keywords Steroids - Sepsis ·

Meta-analysis ·

Trial sequential analysis 


\section{Introduction}

In the intensive care unit (ICU), up to $25 \%$ of admissions are related to sepsis and an additional $12 \%$ of patients develop sepsis during their stay [1]. Sepsis and, if deteriorating, septic shock have a high morbidity and mortality. Depending on the definition of sepsis, mortality varies from 27 to $54 \%$ [1]. To decrease this high rate of morbidity and mortality, several interventions have been suggested. Bundled interventions, like those of the Surviving Sepsis Campaign [2], aim to improve outcome of patients by, among others, early antibiotics, glucocorticosteroids (steroids) and supportive care.

Based on supposed pathophysiological mechanisms, two rationales for steroids in sepsis have been put forward. The first rationale is that high dose steroids may suppress the excess in inflammatory response in sepsis. In the 1970s and the early 1980s high dose steroids (30 mg/ $\mathrm{kg}$ methylprednisolone or equivalent dose) were used in sepsis [3]. In the late 1980s the use declined on the basis of negative results of randomised clinical trials [3]; however, in some centres, high dose steroids are still used in clinical practice today. The second rationale, introduced in the 1990s, is that low dose steroids may recover a relative adrenal insufficiency [4]. Many trials have been conducted, but the pathophysiological basis of the second rationale is still questioned [5]. Possibly both high and low dose steroids can have beneficial or harmful effects in sepsis.

Despite the lack of evidence for the underlying mechanism, the use of low doses of 200-300 mg hydrocortisone is recommended in patients with septic shock not responding to fluid and vasopressor therapy [2]. This recommendation is likely based on results of one systematic review, which found a statistically significant $16 \%$ relative risk reduction (RRR) of mortality (relative risk 0.84; unadjusted $95 \%$ confidence interval 0.72-0.97) in favour of prolonged low dose steroids [6]. The beneficial effect found might be a subgroup effect in more severely ill patients, a spurious finding due to a type I random error as a consequence of repetitive testing as the information size required for showing a $16 \%$ RRR was far from being reached, or an overestimation of a treatment effect due to bias and suboptimal trial methodology [7-12].

A sound methodology in a systematic review is as important as in any type of study to avoid critical errors in the analyses and conclusions [13]. We therefore decided to conduct a new systematic review evaluating the effects of steroids for sepsis in patients with systemic inflammatory response syndrome (SIRS), sepsis, severe sepsis or septic shock as previous meta-analyses fall short on several aspects of rigorous methodology.
Objective

The objective was to perform a systematic review according to a published protocol following guidelines from PRISMA [14] and The Cochrane Handbook for Systematic Reviews of Interventions [15]. We also planned to execute meta-analyses and trial sequential analyses (TSA) of randomised clinical trials that compared the benefits and harms of high and/or low dose steroids for patients with SIRS, sepsis, severe sepsis or septic shock. Our primary outcome was mortality at longest follow-up and serious adverse events.

Available evidence was to be evaluated in the perspective of the three dimensions of possible risks of errors: systematic errors (bias), design errors (also leading to systematic errors due to outcomes, comparators, etc.) and random errors ('the play of chance') [16].

\section{Methods}

The systematic review was conducted following the recommendations of The Cochrane Handbook for Systematic Reviews of Interventions [15] and reported according to the PRISMA statement (www.prisma-statement.org). The protocol was published on PROSPERO (http://www.crd. york.ac.uk/PROSPERO, ID: CRD42013005617).

In addition to an overall analysis including all doses, two separate analyses were conducted of high and low dose steroids. The cut-off value of high and low dose steroids was chosen arbitrarily. High doses were defined as daily doses of more than $500 \mathrm{mg}$ hydrocortisone; low doses were defined as a daily intake of equal or less than $500 \mathrm{mg}$. Other steroids were recalculated into the equivalent hydrocortisone dose [17]. When doses were expressed in milligram per kilogram body weight, doses were calculated assuming a body weight of $75 \mathrm{~kg}$.

\section{Eligibility criteria}

Randomised clinical trials that included adult patients (age $>18$ years) with SIRS, sepsis, severe sepsis or septic shock, or any combinations thereof (Table S1). Trials with patients with SIRS were excluded when SIRS criteria were not explicitly described in their methods section. Trials were also excluded when evaluating steroids for the prevention of the occurrence of SIRS. No limitations were made regarding underlying cause of illness. There were no restrictions on duration of treatment (days) or whether administration occurred continuously or intermittently. All types of steroids were included. 
Trials were included independently of the type of control intervention: placebo, no intervention, or any other control intervention. Co-interventions were allowed provided that similar administration occurred in the intervention groups. Trials were included irrespective of chosen outcomes. No limitations were made on the basis of language or publication status. The following study types were excluded: quasi-randomised studies, observational studies, cross-over studies, and studies comparing different doses or different types of steroids in both trial intervention groups.

\section{Search strategy}

We searched the Cochrane Central Register of Controlled Trials (CENTRAL) in The Cochrane Library, PubMed/ Medline, Embase, Web of Science and Cinahl. We also hand-searched the reference lists of included trials and systematic reviews for further trials. Ongoing trials were sought through trial registries (www.clinicaltrials.gov, www.controlled-trails.com, www.centerwatch.com). No time restrictions were applied. The electronic literature search strategies are listed in Table S2.

\section{Study selection and data extraction}

Two authors independently reviewed all identified titles and abstracts and excluded clearly irrelevant hits. The remaining hits were evaluated in full text. Disagreements were resolved through discussion and the hits excluded on the basis of full text were all listed.

Characteristics of patients and trials and data for analyses were extracted by two authors independently from the included reports. A summary of the recorded patient data is presented in Table 1. A thorough recording of patient data is listed in Table S5.

We contacted corresponding authors for unreported data.

\section{Outcomes}

Primary outcomes were mortality at longest follow-up and serious adverse events. Serious adverse events were a composite outcome, summarizing all serious events excluding mortality, necessitating an intervention, operation or prolonged hospital stay.

Secondary outcomes were persistent dependence on haemodialysis and duration of mechanical ventilation. Additionally, time-specific analyses of mortality at 30 and 90 days were conducted as secondary outcomes according to availability of data. Data of trials reporting 28-day mortality were included in 30-day mortality analyses. All outcomes were classified according to the patients' perspective according to GRADE Working Group (Table S3) [18]. Although used in previous trials and meta-analyses, shock reversal was not considered, as it is a surrogate outcome, which is not important according to the patients' perspective [19].

\section{Risk of bias assessment}

We assessed the risk of bias according to The Cochrane Handbook for Systematic Reviews of Interventions [15], including all eight domains: random sequence generation, allocation concealment, blinding of participants and personnel, blinding of outcome assessment, incomplete outcome data, selective reporting, and other bias (academic or funding bias). If one or more of the domains were judged as having high or unclear risk of bias, the trial was classified as having a high risk of bias. Only two trials had low risk of bias (in all domains) with a pooled information size of 591 patients (nearly all data came from one trial [20]). Therefore, we formulated a group of trials with lower risk of bias. These trials had at least low risk of bias in sequence generation, allocation concealment, blinding of participants and personnel, and blinding of outcome assessment. This does not, however, exclude risk of bias from other domains.

\section{Statistical analysis}

Review Manager 5.1.6 was used for statistical analyses. We used the TSA program version 0.9 beta (www.ctu.dk/tsa; [21]) to control random errors and assess imprecision. For each included trial we calculated the relative risk (RR) with $95 \%$ confidence intervals (CI) for dichotomous outcomes. We report risk differences if statistically significant different from relative risk. We calculated the numbers needed to treat or numbers needed to harm with $95 \%$ CI based on a statistically significant RR.

Heterogeneity among trials was explored by the Chisquared test with significance set at $P$ value of 0.10 , and quantified with inconsistency factor $\left(I^{2}\right)$ statistics. We reported the results from the random-effects model anticipating abundant clinical heterogeneity (in populations, interventions and settings). We reported the results from a fixed-effect model if one or two trials dominated the available evidence [22].

The following subgroup analyses were planned: (1) the stratification of bias risk of trials (lower risk of bias compared to high risk of bias); (2) the duration of steroid treatment [long-term ( $\geq 4$ days) compared to short-term ( $<4$ days) use]; (3) patients with SIRS or sepsis compared to patients with severe sepsis or septic shock; (4) stratification based on the aetiology of sepsis; (5) trials using dexamethasone compared with trials using any other 


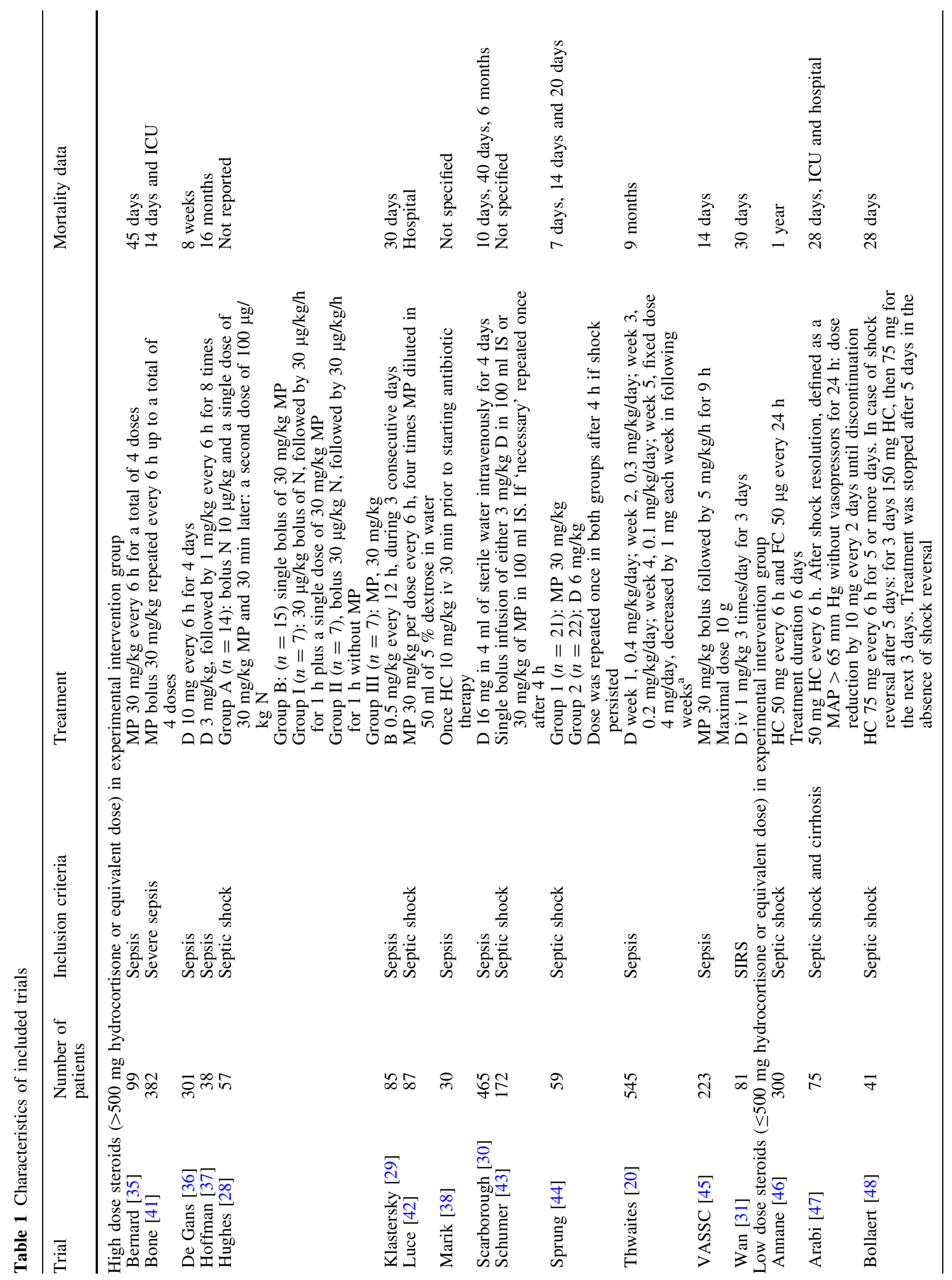




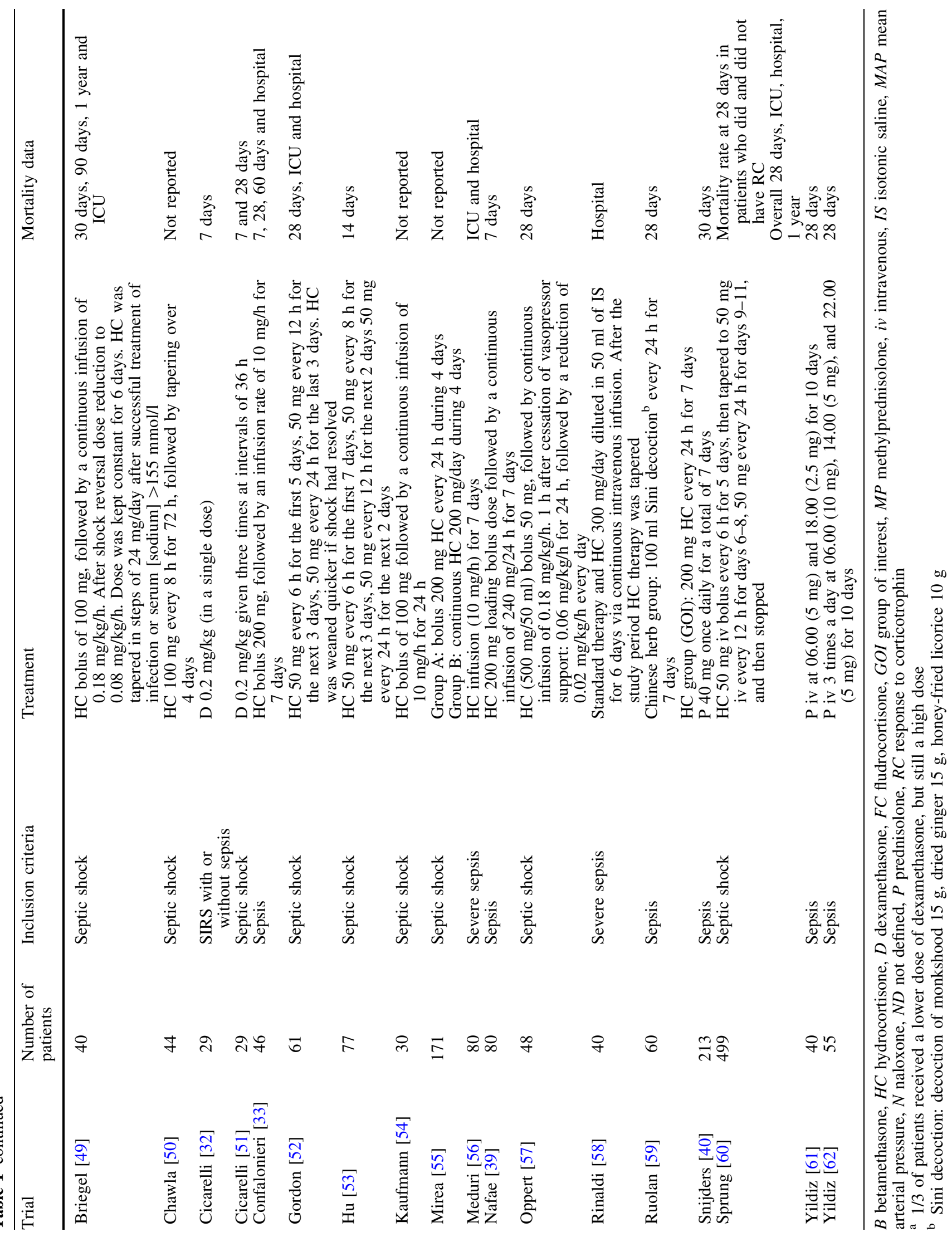


steroid possessing mineralocorticoid properties (unlike dexamethasone).

Trial sequential analysis

We conducted trial sequential analysis (TSA). Conventional meta-analysis runs the risks of random errors due to sparse data and repetitive testing [15, 18]. TSA adjusts the confidence intervals, if data are sparse or repeatedly analysed as a result of multiple updates, to allow firm conclusions. TSA is similar to interim analysis in a single trial where monitoring boundaries are used to decide whether the trial should be terminated early or whether the confidence interval and the adjacent $P$ value are sufficiently narrow or small respectively to show the anticipated effect [23]. In the same manner, trial sequential monitoring boundaries can be applied to metaanalyses [9-12, 24].

TSA depends on the quantification of the required information size. We calculated a diversity-adjusted $\left(D^{2}\right)$ required information size, since the heterogeneity adjustment with $I^{2}$ underestimates the required information size [25]. TSA was conducted with the intention to maintain an overall $5 \%$ risk of a type I error and a power of $90 \%$. For the calculation of the required information size, we anticipated an intervention effect of a $10 \%$ RRR using the control event proportion calculated from the actual meta-analyses. We provided the TSA-adjusted CI for sparse data and repetitive testing, which we described as the trial sequential analysis adjusted CI. We also performed sensitivity analyses with a power of $80 \%$ and assuming a $20 \%$ RRR.

Grade assessment of outcomes

Data on the outcomes of all trials were assessed for the risk of bias measured by the level of evidence, the risk of random error measured by standard error, and the design error measured by grading the outcomes according to GRADE $[16,26]$. Data were presented in a three-dimensional Manhattan error matrix that facilitates the overview of available evidence at a glance and may identify possible lacunae [16]. A GRADE assessment of all outcomes considering risk of bias, inconsistency, imprecision and risk of publication bias was conducted as well [22].

\section{Results}

The search retrieved 5366 hits (Fig. S1). A total of 48 articles were included describing 35 distinct randomised clinical trials. One ongoing trial was identified [27]. Two papers were translated from Chinese by a native Chinese medical doctor. The excluded trials and reasons for exclusion are listed (Table S4).

\section{Characteristics of trials}

Thirty-five randomised trials were included. Fourteen trials evaluated high dose $(>500 \mathrm{mg}$ hydrocortisone or equivalent) steroids and 21 trials used low dose $(\leq 500 \mathrm{mg}$ hydrocortisone or equivalent) steroids (Table 1 and Table S5). Fifteen trials included patients with septic shock and two trials included patients with SIRS. Duration of steroid treatment varied between one single dose and 8 weeks. The daily dose of steroids varied between $30 \mathrm{mg}$ and $600 \mathrm{mg} / \mathrm{kg}$ (total $45 \mathrm{~g}$ ) hydrocortisone (or equivalent) (Fig. 1). In the high dose trials the daily doses varied between $10 \mathrm{mg} / \mathrm{kg}$ (total $750 \mathrm{mg}$ ) and $600 \mathrm{mg} / \mathrm{kg}$ (total $45 \mathrm{~g}$ ) hydrocortisone (or equivalent). In the low dose trials daily doses varied between $30 \mathrm{mg}$ and $440 \mathrm{mg}$ hydrocortisone (or equivalent).

There was insufficient data to evaluate the outcomes persistent dependence on haemodialysis and duration of mechanical ventilation.

\section{Bias risk assessment}

Bias risk of trials was assessed according to The Cochrane Handbook for Systematic Reviews of Interventions (Fig. S2) [15]. Only two trials scored low risk of bias in all domains. Therefore, we used the prespecified group of trials with lower risk of bias in four domains. A total of 16 studies were assessed to have lower risk of bias (Fig. S2).

\section{Effects of interventions}

All pooled intervention effects with their $95 \% \mathrm{CI}$ of all trials along with subgroup effects and all TSA are listed in Table 2.

\section{Comparison 1: steroids versus placebo or no intervention}

Thirty-five trials randomised 4682 patients and evaluated any dose of steroids in patients with sepsis. Two trials had low risk of bias. We considered 16 trials to have lower risk of bias.

All-cause mortality within longest follow-up. Thirty-one trials (including 4290 patients) provided mortality data. Mortality within longest follow-up was $37.6 \%$ in the steroids group and $41.0 \%$ in the control group. Substantial heterogeneity was found $\left(I^{2}=54 \%\right)$. There was 


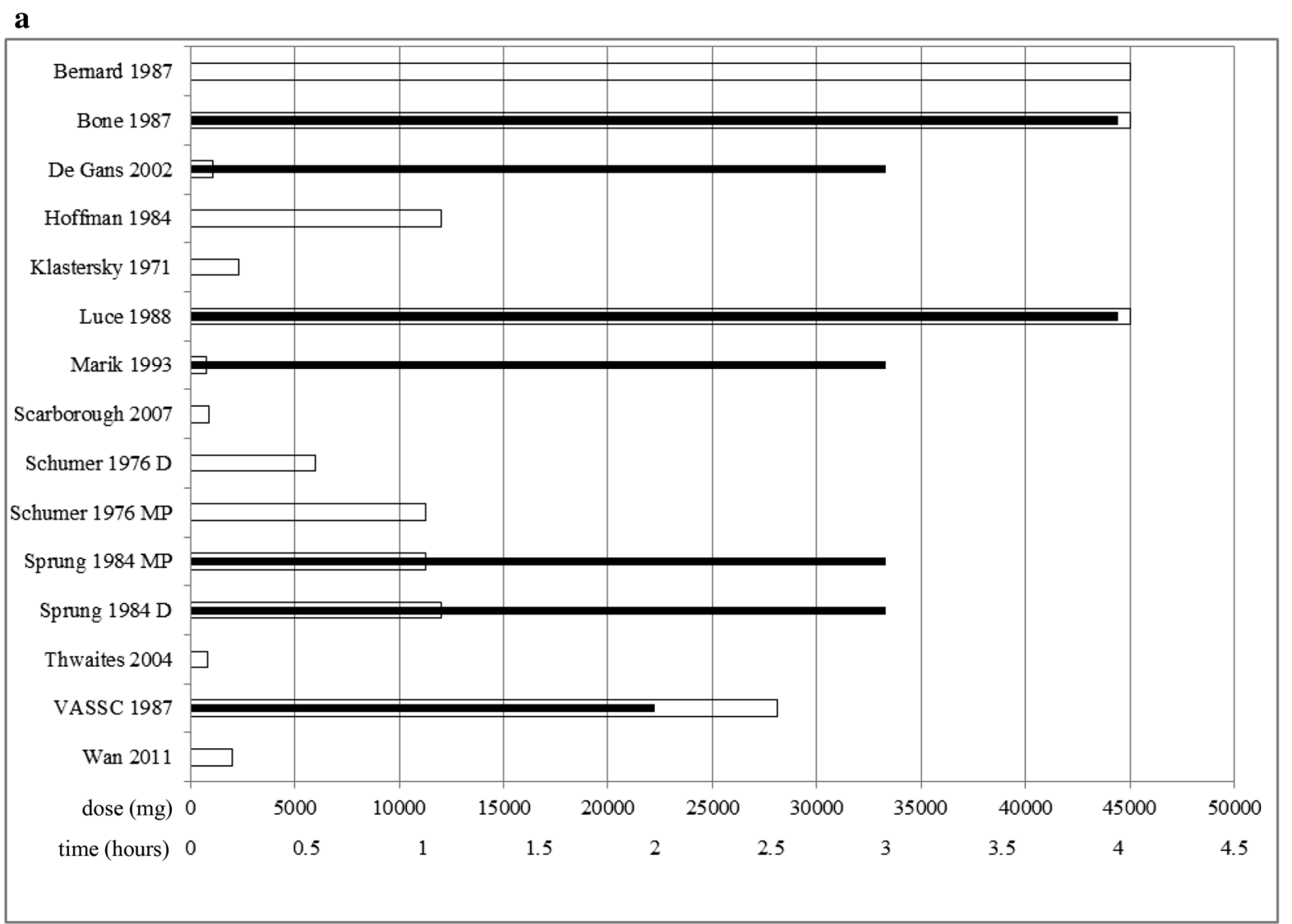

Fig. 1 Randomised clinical trials with mortality data showing for each trial the hydrocortisone dose (on the first day) (white bars) and the time interval from sepsis/septic shock onset until randomisation/start treatment (black bars); a high dose steroids (>500 mg hydrocortisone or equivalent) and b low dose ( $\leq 500 \mathrm{mg}$ hydrocortisone or equivalent). When other steroids were used, the equivalent hydrocortisone dose was calculated using the table in the Oxford Handbook of Critical Care [17]. When doses were

no statistical significant difference (random-effects model RR 0.89, $95 \%$ CI 0.79-1.01; TSA-adjusted CI 0.74-1.08; Figs. 2, S3). TSA on the two low risk of bias trials appeared impossible because of insufficient data; the conventional model showed no statistically significant effect (Table 2). Subgroup analysis based on the prespecified lower risk of bias trials revealed no differential effect. TSA of all trials (RRR $10 \%$; power $90 \%$ ) showed that the accrued and the required information size were far apart (Fig. S3) and that more than 17,000 additional patients may need to be randomised before firm conclusions can be drawn regarding the effect on mortality. However, futility has been reached in our sensitivity trial sequential analysis (RRR $20 \%$; power $80 \%$ ) (Fig. S3) refuting a $20 \%$ RRR to be shown with $80 \%$ power.

Analyses stratified by risk of bias (Fig. 2), treatment duration (long- compared to short-course steroids; Fig. S4), severity of illness (SIRS and sepsis compared to expressed in milligrams per kilogram body weight, daily doses were calculated assuming a body weight of $75 \mathrm{~kg}$. The trials by Hoffman [37], Klastersky [29], Scarborough [30], Schumer [43], Snijders [40], Rinaldi [58], Ruolan [59] and Wan [31] did not provide information on the time interval. The trials by Schumer [43] and Sprung [44] included two intervention groups using different doses of steroids: $D$ dexamethasone, $M P$ methylprednisolone

severe sepsis and septic shock; Fig. S5) and type of steroids (excluding trials using dexamethasone) all showed no statistically significant effects (Table 2) and were in line with the other analyses suggesting that many more patients need to be randomised before firm conclusions may be drawn.

Serious adverse events excluding mortality. No statistical difference was found in serious adverse events including all trials that evaluated steroids for sepsis (random-effects model RR 1.02, $95 \%$ CI 0.92-1.15; TSA-adjusted CI 0.7-1.48; Fig. 3, S6). TSA showed that nearly 50,000 additional patients may need to be randomised before firm conclusions can be drawn on the effect on serious adverse events (Fig. S6). The types of serious adverse events are listed in Table S6. The incidence of serious adverse events did not vary according to the degree of sepsis (Table 2). 
b

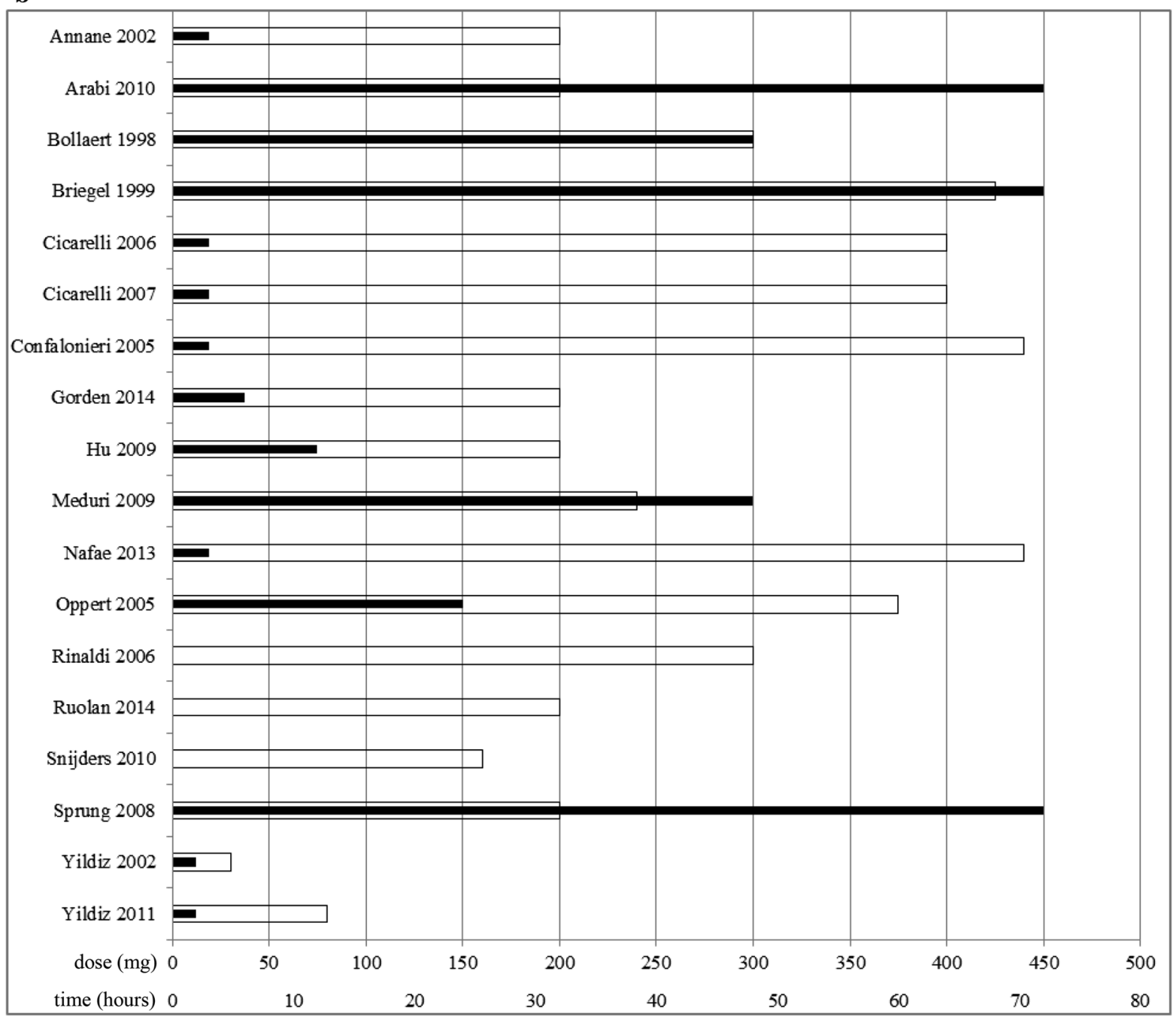

Fig. 1 continued

Other outcomes. Time-specific analyses of mortality were conducted for 30-day (16 trials) and 90-day (two trials) follow-up. We found no statistically significant treatment effect when evaluating 30-day mortality (random-effects model RR 0.96, $95 \%$ CI 0.85-1.08; TSA RR 0.98 , TSA-adjusted CI $0.83-1.17$; point estimates were different as a result of different handling of zero event trials) (Fig. S7). Data for 90-day mortality were too sparse to perform TSA-adjusted analysis; in a conventional analysis no statistically significant effect was found (random-effects model RR 0.36, $95 \%$ CI 0.04-2.90) (Fig. S8).

GRADE assessment considering risk of bias, inconsistency, imprecision and risk of publication bias showed very low quality of evidence (Table S7).
Comparison 2: high dose steroids versus placebo or no intervention

Fourteen trials (2624 patients) evaluated high doses (>500 mg hydrocortisone or equivalent) of steroids for sepsis. Only one trial had low risk of bias in all domains [20]. Six trials were considered to have lower risk of bias (lower risk of bias in four domains). One trial did not report mortality (Table 1) [28].

All-cause mortality within longest follow-up. Six trials with lower risk of bias and seven trials with high risk of bias evaluated mortality in 2537 patients at different lengths of follow-up. No statistically significant beneficial effect from steroid treatment was found (random-effects 


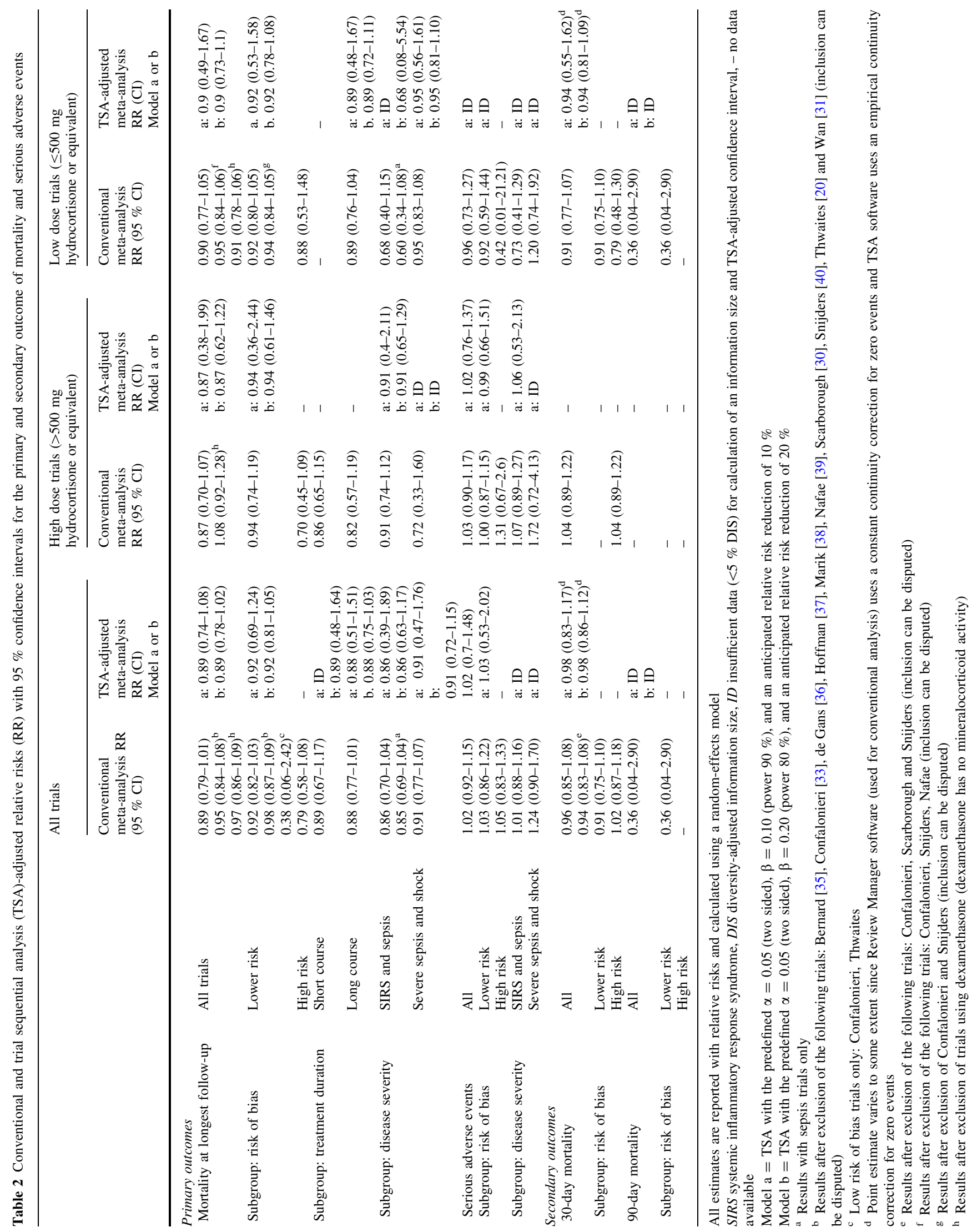




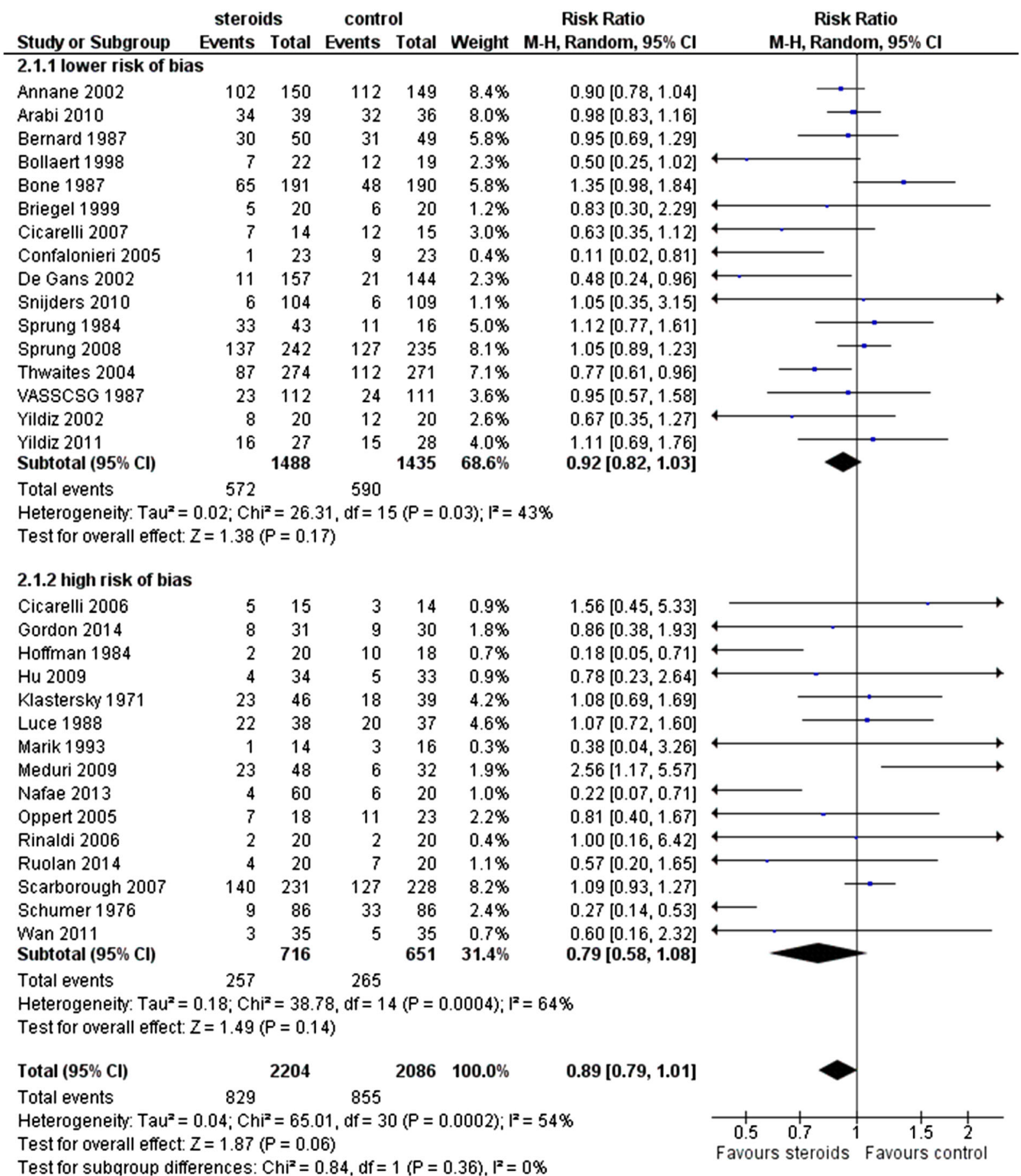

Fig. 2 Forest plot of mortality at longest follow-up of all trials evaluating steroids for sepsis with subgroups according to risk of bias (random-effects model)

model RR 0.87, $95 \%$ CI 0.70-1.07; TSA-adjusted CI trials using dexamethasone) all showed no statistically $0.38-1.99$; Fig. S9).

Analyses stratified by risk of bias (Fig. S9), severity of illness (SIRS and sepsis compared to severe sepsis and Serious adverse events excluding mortality. No statistiseptic shock; Fig. S10) and type of steroids (excluding cal significant difference was found in the overall 


\begin{tabular}{|c|c|c|c|c|c|c|c|c|c|c|}
\hline \multirow[b]{2}{*}{ Study or Subgroup } & \multicolumn{2}{|c|}{ steroids } & \multicolumn{2}{|c|}{ control } & \multirow[b]{2}{*}{ Weight } & $\begin{array}{l}\text { Risk Ratio } \\
\text {. }\end{array}$ & \multirow{2}{*}{\multicolumn{4}{|c|}{$\begin{array}{c}\text { Risk Ratio } \\
\text { M-H, Random, } 95 \% \mathrm{Cl}\end{array}$}} \\
\hline & Events & Total & Events & Total & & M-H, Random, $95 \% \mathrm{Cl}$ & & & & \\
\hline \multicolumn{11}{|c|}{ 3.1.1 lower risk of bias } \\
\hline Annane 2002 & 44 & 150 & 45 & 149 & $7.2 \%$ & $0.97[0.69,1.38]$ & & & 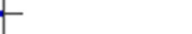 & \\
\hline Arabi 2010 & 25 & 39 & 14 & 36 & $4.5 \%$ & $1.65[1.03,2.64]$ & & & & \\
\hline Bernard 1987 & 8 & 50 & 5 & 49 & $1.1 \%$ & $1.57[0.55,4.46]$ & & & & \\
\hline Cicarelli 2007 & 0 & 14 & 1 & 15 & $0.1 \%$ & $0.36[0.02,8.07]$ & & & & \\
\hline Confalonieri 2005 & 6 & 23 & 18 & 23 & $2.2 \%$ & $0.33[0.16,0.69]$ & & & & \\
\hline De Gans 2002 & 66 & 157 & 50 & 144 & $9.1 \%$ & $1.21[0.91,1.62]$ & & & & \\
\hline Snijders 2010 & 41 & 104 & 37 & 109 & $7.0 \%$ & $1.16[0.82,1.65]$ & & & & \\
\hline Sprung 1984 & 16 & 43 & 3 & 16 & $1.0 \%$ & $1.98[0.67,5.91]$ & & & & \\
\hline Thwaites 2004 & 160 & 274 & 169 & 271 & $17.4 \%$ & $0.94[0.82,1.07]$ & & & & \\
\hline VASSCSG 1987 & 74 & 112 & 79 & 111 & $14.7 \%$ & $0.93[0.78,1.11]$ & & & & \\
\hline Yildiz 2002 & 0 & 20 & 1 & 20 & $0.1 \%$ & $0.33[0.01,7.72]$ & & & & \\
\hline Yildiz 2011 & 0 & 27 & 0 & 28 & & Not estimable & & & & \\
\hline Subtotal $(95 \% \mathrm{Cl})$ & & 1013 & & 971 & $64.5 \%$ & $1.03[0.86,1.22]$ & & & & \\
\hline Total events & 440 & & 422 & & & & & & & \\
\hline \multirow{2}{*}{\multicolumn{11}{|c|}{$\begin{array}{l}\text { Heterogeneity: Tau }{ }^{2}=0.03 ; \mathrm{Chi}^{2}=20.36, \mathrm{df}=10(P=0.03) ;\left.\right|^{2}=51 \% \\
\text { Test for overall effect: } Z=0.30(\mathrm{P}=0.76)\end{array}$}} \\
\hline & & & & & & & & & & \\
\hline \multicolumn{11}{|l|}{ 3.1.2 high risk of bias } \\
\hline Cicarelli 2006 & 0 & 15 & 3 & 14 & $0.2 \%$ & $0.13[0.01,2.38]$ & $\longleftarrow$ & & & \\
\hline Klastersky 1971 & 7 & 46 & 1 & 39 & $0.3 \%$ & $5.93[0.76,46.17]$ & & & & \\
\hline Nafae 2013 & 59 & 60 & 20 & 20 & $21.1 \%$ & $1.00[0.92,1.08]$ & & & & \\
\hline Scarborough 2007 & 109 & 233 & 101 & 232 & $13.4 \%$ & $1.07[0.88,1.31]$ & & & + & \\
\hline Schumer 1976 & 4 & 86 & 3 & 86 & $0.6 \%$ & $1.33[0.31,5.78]$ & & & & \\
\hline Subtotal (95\% Cl) & & 440 & & 391 & $35.5 \%$ & $1.05[0.83,1.33]$ & & & & \\
\hline Total events & 179 & & 128 & & & & & & & \\
\hline \multirow{2}{*}{\multicolumn{11}{|c|}{$\begin{array}{l}\text { Heterogeneity: } \operatorname{Tau}^{2}=0.03 ; \mathrm{Chi}^{2}=8.33, \mathrm{df}=4(P=0.08) ; \mathrm{I}^{2}=52 \% \\
\text { Test for overall effect: } Z=0.38(P=0.70)\end{array}$}} \\
\hline & & & & & & & & & & \\
\hline Total (95\% Cl) & & 1453 & & 1362 & $100.0 \%$ & $1.02[0.92,1.15]$ & & & 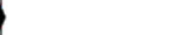 & \\
\hline Total events & 619 & & 550 & & & & & & & \\
\hline Heterogeneity: Tau² & $0.01 ; \mathrm{Chi}^{2}$ & $=26.2$ & 24, $d f=1$ & $5(P=0$ & $0.04) ; 1^{2}=$ & $43 \%$ & 0.01 & $0.1 \quad 1$ & $1 \quad 10$ & 100 \\
\hline Test for subgroup di & $\begin{array}{l}Z=0.43(1) \\
\text { erences: } 0\end{array}$ & $C h i^{2}=0$ & $0.02 \mathrm{df}$ & 1 ( $\mathrm{P}$ & $0.90)$ । & & & Favours steroids & Favours control & \\
\hline
\end{tabular}

Fig. 3 Forest plot of serious adverse events of all trials evaluating steroids for sepsis with subgroups according to risk of bias (randomeffects model)

proportion of serious adverse events (random-effects model RR 1.03, $95 \%$ CI 0.90-1.17; TSA-adjusted RR 1.02 , CI $0.70-1.48)$. No statistically significant differences were found in subgroups according to bias risk and disease severity (Fig. S11, Table 2).

Other outcomes. Three trials with high risk of bias [2931 ] reported mortality at 30 days; no 90-day follow-up data was reported (Table 2). There was no statistically significant effect.

Error matrix plots were constructed for overview of all available evidence for high dose steroids at a glance (Fig. S17). GRADE assessment considering risk of bias, inconsistency, imprecision and risk of publication bias showed very low quality of evidence for all outcomes (Table S8).

\section{Comparison 3: low dose steroids versus placebo or no intervention}

Twenty-one trials randomised 2058 patients for low dose ( $\leq 500 \mathrm{mg}$ hydrocortisone or equivalent) steroids for sepsis. There were large differences between the trials in the time interval between sepsis onset and initiation of steroids treatment: $2-72 \mathrm{~h}$ (Fig. 1). Only one trial applied short-course low dose steroids [32].

All-cause mortality within longest follow-up. Only one trial had low risk of bias in all domains [33]. Ten trials with lower risk of bias (1315 patients) and eight trials with high risk of bias (438 patients) evaluated mortality at different lengths of follow-up. The overall pooled estimate showed no statistical difference (random-effects model RR 0.90, $95 \%$ CI 0.77-1.05; TSA-adjusted CI 0.49-1.67) (Figs. 4, S12).

Analyses stratified by risk of bias (Figs. 4, S12), treatment duration (long- compared to short-course steroids; Fig. S13), severity of illness (SIRS and sepsis compared to severe sepsis and septic shock; Fig. S14) and type of steroids (excluding trials using dexamethasone) all showed no statistically significant effects (Table 2). Sensitivity analyses (TSA, RRR $20 \%$; power $80 \%$ ) based on lower risk of bias trials and separately all trials evaluating severe sepsis and septic shock showed futility for treatment with low dose steroids (Figs. S12, S14). 


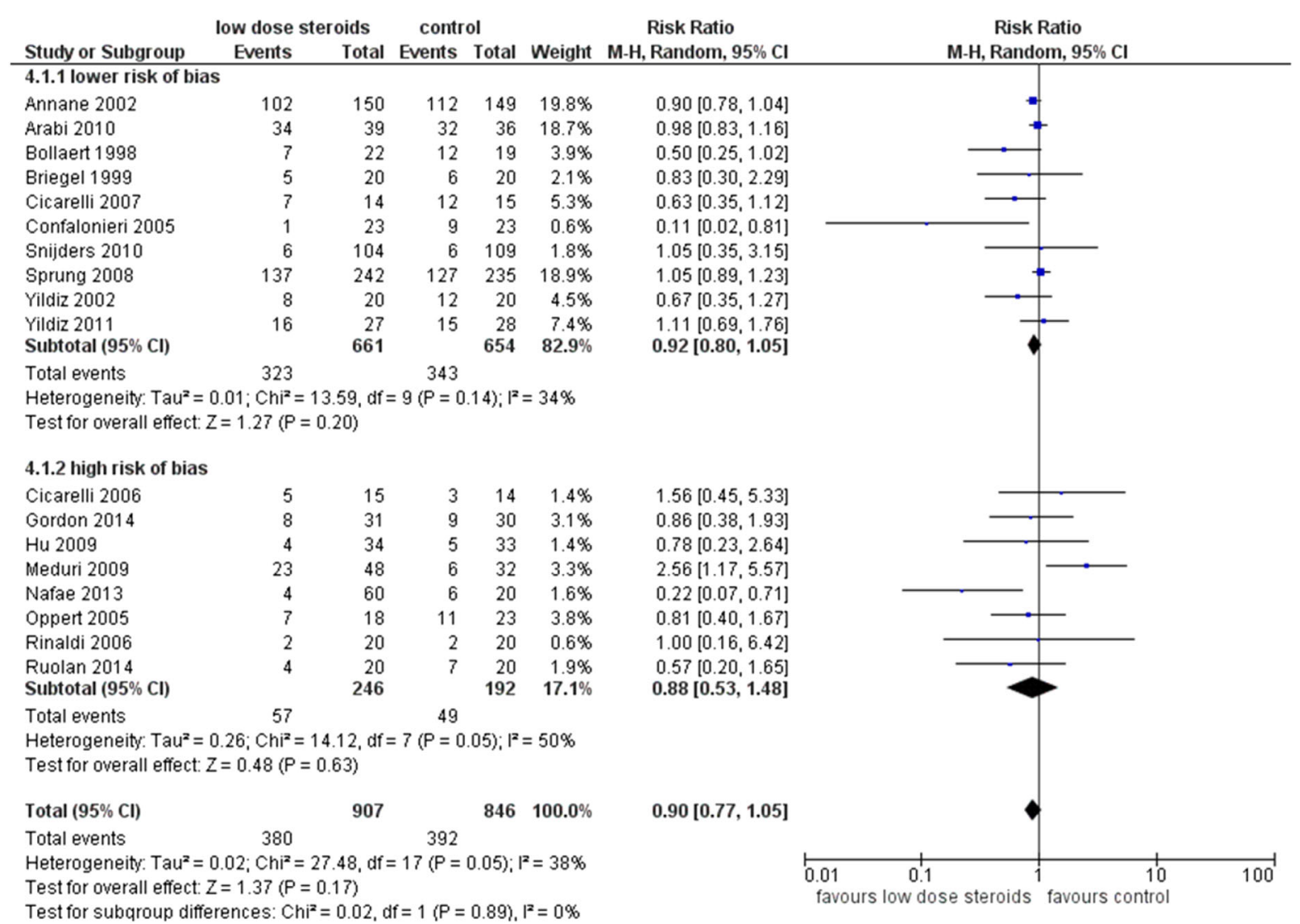

Fig. 4 Forest plot of mortality at longest follow-up of low dose steroids ( $\leq 500 \mathrm{mg}$ hydrocortisone or equivalent) use according to risk of bias subgroups (random-effects model)

Serious adverse events excluding mortality. Nine trials (with 866 patients) evaluated serious adverse events. One trial had low risk of bias [33]. Seven trials had lower risk of bias. Data were too sparse to perform a TSA. A conventional analysis found no statistically significant intervention effect (random-effects model RR 0.96, $95 \%$ CI 0.73-1.27; Fig. S15). The incidence of serious adverse events did not vary according to the degree of sepsis (Table 2).

Mortality at 30 days. Thirteen trials (1479 patients) evaluated mortality at 30-day follow-up. One trial had low risk of bias [33]. Ten trials had lower risk of bias. No significant benefit was found from steroids treatment (random-effects model RR 0.91, $95 \%$ CI 0.77-1.07; TSA RR 0.94, TSA-adjusted CI $0.55-1.62$; point estimates were different as a result of different handling of zero event trials; Fig. S16). Subgroup analysis based on bias risk showed no differential effect. TSA estimated that many more randomized patients are needed before firm conclusions can be drawn. A sensitivity analysis (TSA, RRR $20 \%$; power $80 \%$ ) showed futility for low dose steroids treatment (Fig. S16).
Mortality at 90 days. The results of 90-day mortality in low dose steroids are equal to the overall 90-day mortality, since only trials that evaluated low dose steroids provided data on 90-day follow-up.

Other outcomes. Error matrix plots were constructed for overview of all available evidence for low dose steroids at a glance (Fig. S18). Data suggest that there are considerable risks of systematic errors (bias) and random errors (large standard errors on average). GRADE assessment considering risk of bias, inconsistency, imprecision and risk of publication bias showed very low quality of evidence for all outcomes (Table S9).

\section{Discussion}

We did not find evidence for a beneficial effect of an intervention with steroids in patients with SIRS, sepsis, severe sepsis or septic shock in this systematic review with meta-analyses and TSA, including 35 randomised trials with 4682 patients. High $(>500 \mathrm{mg}$ hydrocortisone 
or equivalent) and low dose ( $\leq 500 \mathrm{mg}$ hydrocortisone or equivalent) steroids were evaluated in separate comparisons and no evidence of a beneficial effect was found. Moreover, TSA suggested that more than 17,000 patients need to be randomised before firm conclusions can be drawn on any present or absent intervention effect with a $10 \%$ RRR. Evidence has been reached to refute a $20 \%$ RRR with a power of $80 \%$.

Our conclusion contrasts with previous publications suggesting beneficial effects associated with use of a long course of low dose steroids [6]. Differences might be explained by another search strategy and different analyses with improved accounting for risks of systematic, design and random errors. There is accumulating evidence that random error plays an important role in premature conclusions of spurious significant findings [11]. In simulation studies up to $30 \%$ of premature declarations of significant effects are in fact overestimations of intervention effects, once sufficient evidence has been reached [34].

A substantial clinical heterogeneity existed between the included trials, even after separation into high and low doses of steroids. Within both high and low dose steroid groups the administrated daily dose of steroids differed importantly (Fig. 1). Moreover, there were substantial differences regarding the time interval between sepsis onset and administration of the first steroid dose, e.g. in antibiotic therapy timing appears to be an essential feature for any beneficial effect. Therefore, lack of both optimised dosing and optimised timing might obscure potential beneficial effects of steroids. As dexamethasone has no mineralocorticoid activity, analyses were repeated excluding trials using dexamethasone and results appeared to be similar (Table 2).

Retrospectively, our study protocol could have been more specific on exclusion criteria for trials evaluating patients with SIRS and sepsis. We excluded trials that evaluated steroids for prevention of diseases and for autoimmune diseases. We also excluded trials that evaluated steroids for treatment of localized oedema. Furthermore, we only included trials that evaluated patients with SIRS if the criteria for SIRS were explicitly stated in the methods section of the report; however, SIRS criteria may not predict patient important outcomes and may be too inclusive. Although we reached consensus through discussions, the inclusion of some trials can still be discussed $[20,30,31,33,35-40]$. To test the robustness of our conclusions, we therefore conducted several sensitivity analyses by excluding these trials and they all resulted in similar findings (Table 2).

Trial sequential analyses, an error matrix to evaluate risks of errors and GRADE assessment add to the strength of the conclusions. However, our systematic review mirrors the lack of quality and quantity of the included randomised clinical trials. The included trials fall short on bias protection, included numbers of patients and chosen outcomes. Therefore, the evidence of steroids for sepsis (both high and low dose) is characterized by high risks of both systematic errors and random errors. We used wide inclusion criteria to evaluate the effect of steroids in the broad spectrum of disease. Although none of the analyses showed a statistically significant effect, we cannot exclude a small beneficial intervention effect of steroids in a specific subgroup.

\section{Conclusion}

In this systematic review with meta-analyses and TSA we did not find any statistically significant beneficial effect that could support propagating the use of steroids for sepsis. Further, our TSA suggests that many thousands of randomised patients are needed in order to change this perspective. Therefore, steroids should no longer be recommended for patients with sepsis outside ongoing [27] or future well-designed randomised clinical trials with low risks of both systematic error and random error.

Acknowledgments We thank Y. J. Gu for translation of the Chinese papers and $\mathrm{W}$. Bruins for language suggestions. We also thank Dr. Confalonieri for providing additional unpublished follow-up data.

Conflicts of interest None of the authors has a conflict of interest to declare.

Open Access This article is distributed under the terms of the Creative Commons Attribution-NonCommercial 4.0 International License (http://creativecommons.org/licenses/by-nc/4.0/), which permits any noncommercial use, distribution, and reproduction in any medium, provided you give appropriate credit to the original author(s) and the source, provide a link to the Creative Commons license, and indicate if changes were made.

\section{References}

1. Vincent JL, Sakr Y, Sprung CL et al (2006) Sepsis in European intensive care units: results of the SOAP study. Crit Care Med 34:344-353
2. Dellinger RP, Levy MM, Rhodes A et al (2013) Surviving Sepsis Campaign: international guidelines for management of severe sepsis and septic shock, 2012. Intensive Care Med 39:165-228
3. Batzofin BM, Sprung CL, Weiss YG (2011) The use of steroids in the treatment of severe sepsis and septic shock. Best Pract Res Clin Endocrinol Metab 25:735-743 
4. Annane D, Bellissant E, Sebille V, Lesieur O, Mathieu B, Raphael JC, Gajdos P (1998) Impaired pressor sensitivity to noradrenaline in septic shock patients with and without impaired adrenal function reserve. $\mathrm{Br} \mathbf{J}$ Clin Pharmacol 46:589-597

5. Venkatesh B, Cohen J (2011) Adrenocortical (dys)function in septic shock - a sick euadrenal state. Best Pract Res Clin Endocrinol Metab 25:719-733

6. Annane D, Bellissant E, Bollaert PE et al (2009) Corticosteroids in the treatment of severe sepsis and septic shock in adults: a systematic review. JAMA 301:2362-2375

7. Turner R, Bird S, Higgins J (2013) The impact of study size on meta-analyses: examination of underpowered studies in cochrane reviews. PLoS ONE 8(3):e59202

8. Higgins JP, Whitehead A, Simmonds M (2011) Sequential methods for randomeffects meta-analysis. Stat Med 30:903-921

9. Pogue JM, Yusuf S (1997) Cumulating evidence from randomized trials utilizing sequential monitoring boundaries for cumulative metaanalysis. Control Clin Trials 18:580-593 (discussion 661-6)

10. Pogue J, Yusuf S (1998) Overcoming the limitations of current meta-analysis of randomised controlled trials. Lancet 351:47-52

11. Brok J, Thorlund K, Wetterslev J, Gluud C (2009) Apparently conclusive meta-analyses may be inconclusivetrial sequential analysis adjustment of random error risk due to repetitive testing of accumulating data in apparently conclusive neonatal metaanalyses. Int J Epidemiol 38:287-298

12. Wetterslev J, Thorlund K, Brok J, Gluud C (2008) Trial sequential analysis may establish when firm evidence is reached in cumulative metaanalysis. J Clin Epidemiol 61:64-75

13. da Costa BR (2014) Systematic reviews and meta-analyses of randomized trials: principles and pitfalls. Eur Heart $\mathbf{J}$ 35:3336-3345

14. Shamseer L (2015) Preferred reporting items for systematic review and metaanalysis protocols (PRISMA-P) 2015: elaboration and explanation. BMJ 349:g7647

15. Higgins JPT, Green S (2011) Cochrane handbook for systematic reviews of interventions version 5.1.0 [updated March 2011]. The Cochrane Collaboration. www.cochrane-handbook.org
16. Keus F, Wetterslev J, Gluud C, van Laarhoven CJ (2010) Evidence at a glance: error matrix approach for overviewing available evidence. BMC Med Res Methodol 10:90

17. Singer M, Webb R (eds) (2009) Oxford handbook of critical care, 3rd edn. Oxford University Press, Oxford, p 329

18. Guyatt GH, Oxman AD, Kunz R, Vist GE, Falck-Ytter Y, Schunemann HJ, GRADE Working Group (2008) What is "quality of evidence" and why is it important to clinicians? BMJ 336:995-998

19. Gluud C (2007) Hepatology may have problems with putative surrogate outcome measures. J Hepatol 46:734-742

20. Thwaites GE (2004) Dexamethasone for the treatment of tuberculous meningitis in adolescents and adults. N Engl J Med 351:1741-1751

21. Thorlund K, Engstrom J, Wetterslew J, Brok J, Imberger G, Gluud C (2011) User manual for trial sequential analysis (TSA). Copenhagen Trial Unit, Centre for Clinical Intervention Research, Copenhagen, Denmark, pp 1-115. www.ctu.dk/tsa

22. Jakobsen JC (2014) Thresholds for statistical and clinical significance in systematic reviews with meta-analytic methods. BMC Med Res Methodol $14: 120$

23. Jakobsen JC, Gluud C, Winkel P, Lange T, Wetterslev J (2014) The thresholds for statistical and clinical significancea five-step procedure for evaluation of intervention effects in randomised clinical trials. BMC Med Res Methodol $14: 34$

24. Thorlund K, Devereaux PJ, Wetterslev J et al (2009) Can trial sequential monitoring boundaries reduce spurious inferences from meta-analyses? Int J Epidemiol 38:276-286

25. Wetterslev J, Thorlund K, Brok J, Gluud C (2009) Estimating required information size by quantifying diversity in random-effects model metaanalyses. BMC Med Res Methodol 9:86

26. Guyatt GH, Oxman AD, Vist GE et al (2008) GRADE: an emerging consensus on rating quality of evidence and strength of recommendations. BMJ 336:924-926

27. Venkatesh B, Myburgh J, Finfer S et al (2013) The ADRENAL study protocol: adjunctive corticosteroid treatment in critically ill patients with septic shock. Crit Care Resusc 15:83-88

28. Hughes GS Jr (1984) Naloxone and methylprednisolone sodium succinate enhance sympathomedullary discharge in patients with septic shock. Life Sci 35:2319-2326
29. Klastersky J, Cappel R, Debusscher L (1971) Effectiveness of betamethasone in management of severe infections. A double-blind study. N Engl J Med 284:1248-1250

30. Scarborough M (2007) Corticosteroids for bacterial meningitis in adults in subSaharan Africa. N Engl J Med 357:2441-2450

31. Wan MH (2011) Clinical observation on the effect of dexamethasone and Chinese herbal decoction for purgation in severe acute pancreatitis patients. Chin J Integr Med 17:141-145

32. Cicarelli DD, Bensenor FE, Vieira JE (2006) Effects of single dose of dexamethasone on patients with systemic inflammatory response. Sao Paulo Med J 124:90-95

33. Confalonieri M, Urbino R, Potena A et al (2005) Hydrocortisone infusion for severe community-acquired pneumonia: a preliminary randomized study. Am J Respir Crit Care Med 171:242-248

34. Thorlund $\mathrm{K}$, Imberger $\mathrm{G}$, Walsh $\mathrm{M}$ et al (2011) The number of patients and events required to limit the risk of overestimation of intervention effects in meta-analysis - a simulation study. PLoS One 6:e25491

35. Bernard GR, Luce JM, Sprung CL et al (1987) High-dose corticosteroids in patients with the adult respiratory distress syndrome. N Engl J Med 317:1565-1570

36. de Gans J (2002) Dexamethasone in adults with bacterial meningitis. N Engl J Med 347:1549-1556

37. Hoffman SL (1984) Effective treatment and prevention of typhoid fever: updated. Trans Am Clin Climatol Assoc 95:52-65

38. Marik PP (1993) Hydrocortisone and tumor necrosis factor in severe community-acquired pneumonia. A randomized controlled study. Chest 104:389-392

39. Nafae R, Ragab M, Amany F, Rashed S (2013) Adjuvant role ofcorticosteroids in the treatment of communityacquiredpneumonia. Egypt J Chest Dis Tuberc 62:439-445

40. Snijders D (2010) Efficacy of corticosteroids in community-acquired pneumonia: a randomized doubleblinded clinical trial. Am J Respir Crit Care Med 181:975-982

41. Bone RC, Fisher CJ Jr, Clemmer TP, Slotman GJ, Metz CA, Balk RA (1987) A controlled clinical trial of high-dose methylprednisolone in the treatment of severe sepsis and septic shock. N Engl J Med 317:653-658 
42. Luce JM, Montgomery AB, Marks JD, Turner J, Metz CA, Murray JF (1988) Ineffectiveness of high-dose methylprednisolone in preventing parenchymal lung injury and improving mortality in patients with septic shock. Am Rev Respir Dis 138:62-68

43. Schumer W (1976) Steroids in the treatment of clinical septic shock. Ann Surg 184:333-341

44. Sprung CL, Caralis PV, Marcial EH et al (1984) The effects of high-dose corticosteroids in patients with septic shock. A prospective, controlled study. N Engl J Med 311:1137-1143

45. Veterans Administration Systemic Sepsis Cooperative Study Group (1987) Effect of high-dose glucocorticoid therapy on mortality in patients with clinical signs of systemic sepsis. The Veterans Administration Systemic Sepsis Cooperative Study Group. N Engl J Med 317:659-665

46. Annane D, Sebille V, Charpentier C et al (2002) Effect of treatment with low doses of hydrocortisone and fludrocortisone on mortality in patients with septic shock. JAMA 288:862-871

47. Arabi YM, Aljumah A, Dabbagh O et al (2010) Low-dose hydrocortisone in patients with cirrhosis and septic shock: a randomized controlled trial. CMAJ 182:1971-1977

48. Bollaert PE, Charpentier C, Levy B, Debouverie M, Audibert G, Larcan A (1998) Reversal of late septic shock with supraphysiologic doses of hydrocortisone. Crit Care Med 26:645-650

49. Briegel J, Forst H, Haller M et al (1999) Stress doses of hydrocortisone reverse hyperdynamic septic shock: a prospective, randomized, double-blind, single-center study. Crit Care Med 27:723-732
50. Chawla K, Kupfer Y, Tessler S (1999) Hydrocortisone reverses refractory septic shock [abstract]. Crit Care Med 27:A33

51. Cicarelli DD, Vieira JE, Bensenor FE (2007) Early dexamethasone treatment for septic shock patients: a prospective randomized clinical trial. Sao Paulo Med J 125:237-241

52. Gordon AC, Mason AJ, Perkins GD, Stotz M, Terblanche M, Ashby D, Brett SJ (2014) The interaction of vasopressin and corticosteroids in septic shock: a pilot randomized controlled trial. Crit Care Med 42:1325-1333

53. Hu B, Li JG, Liang H et al (2009) The effect of low-dose hydrocortisone on requirement of norepinephrine and lactate clearance in patients with refractory septic shock. Zhongguo Wei Zhong Bing Ji Jiu Yi Xue 21:529-531

54. Kaufmann I, Briegel J, Schliephake F et al (2008) Stress doses of hydrocortisone in septic shock: beneficial effects on opsonizationdependent neutrophil functions. Intensive Care Med 34:344-349

55. Mirea L, Ungureanu R, Pavelescu D, Grintescu I, Dumitrache C, Mirea D, Grintescu I (2014) Continuous administration of corticosteroids in septic shock can reduce risk of hypernatremia. Critical care (London, England) conference: 34th international symposium on intensive care and emergency medicine, Brussels, Belgium. Conference Publication, S86
56. Meduri GU, Golden E, Umberger R (2009) Prospective double-blind randomized clinical trial on the effects of low-dose hydrocortisone infusion in patients with severe sepsis. Chest 136:45S

57. Oppert M, Schindler R, Husung C et al (2005) Low-dose hydrocortisone improves shock reversal and reduces cytokine levels in early hyperdynamic septic shock. Crit Care Med 33:2457-2464

58. Rinaldi S, Adembri C, Grechi S, De Gaudio AR (2006) Low-dose hydrocortisone during severe sepsis: effects on microalbuminuria. Crit Care Med 34:2334-2339

59. Ruolan H, Zhong Z, Mujuan X, Xiao C, Qiujie Q, Ling W, Xinke M (2014) Effect of Sini decoction on function of hypothalamic-pituitary-adrenal axis in patients with sepsis. Zhonghua Wei Zhong Bing Ji Jiu Yi Xue 26:184 (in Chinese)

60. Sprung CL, Annane D, Keh D et al (2008) Hydrocortisone therapy for patients with septic shock. N Engl J Med 358:111-124

61. Yildiz O, Doganay M, Aygen B, Guven M, Kelestimur F, Tutuu A (2002) Physiological-dose steroid therapy in sepsis. Crit Care 6:251-259

62. Yildiz O, Tanriverdi F, Simsek S, Aygen B, Kelestimur F (2011) The effects of moderate-dose steroid therapy in sepsis: a placebo-controlled, randomized study. J Res Med Sci 16:1410-1421 